\title{
Positron Emission Tomography Plays a More Important Role in Health Care
}

\section{Guohui Wang*}

Department of Internal Medicine, The Affiliated Tumor Hospital of Zhengzhou University, Zhengzhou 450003, China

Corresponding author: Guohui Wang, Department of Internal Medicine, The Affiliated Tumor Hospital of Zhengzhou University, Zhengzhou 450003, China, Tel: 1-832-289-943; E-mail: wgh3001@qq.com

Received date: Apr 04, 2015; Accepted date: Apr 15, 2015; Published date: Apr 22, 2015

Copyright: (c) 2015 Guohui Wang. This is an open-access article distributed under the terms of the Creative Commons Attribution License, which permits unrestricted use, distribution, and reproduction in any medium, provided the original author and source are credited.

\section{Mini review}

Positron emission tomography (PET) [1] scanning, a functional imaging technique, is playing a more and more important role in modern medical science compared to the conventional anatomy imaging technologies such as X-ray and computed tomography (CT), ultrasound, and magnetic resonance imaging (MRI). Though with the help of modern computer technology, the CT, MRI, and ultrasound have begun to be used for functional imaging, the PET scan still has advantages over other imaging modalities.

In the late 1950s, David E. Kuhlet et al., introduced the concept of emission and transmission tomography. Later, their work led to the design and construction of several tomographic instruments at the University of Pennsylvania. Tomographic imaging techniques were further developed by Michel Ter-Pogossian et al. at Washington University School of Medicine [2-3].

The PET system detects pairs of gamma rays emitted indirectly by a positron-emitting radionuclide, which is introduced into the body on a biologically active molecule. In the early stages, there was only a PET detector in the system, which could not accurately locate the site of the foci in the body. In order to address this pitfall of the PET scanner, the combined PET-CTsystem was developed. This scanner could accomplish a CT X-ray scan and a PET scan on the patient during the same session, in the same machine. This combined PET-CT scanner could give both anatomic and metabolic information about a lesion, which greatly increased the accuracy rate of diagnoses of the radiologist.

At present, the radionuclides used in PET scanning are short halflife radionuclides, such as carbon-11 $(\sim 20 \mathrm{~min})$, nitrogen-13 $(\sim 10$ min), oxygen-15 ( 2 min), fluorine-18 ( 110 min), gallium-68 ( 67 $\mathrm{min})$, or rubidium-82( 1.27 $\mathrm{min})$. These radionuclides are incorporated either into compounds normally used by the body such as glucose (or glucose analogues), water, or ammonia, or into molecules that bind to receptors or other sites of drug action, called radiotracers.

PET-CT is used heavily in clinical oncology for the primary diagnosis and staging, evaluation of the treatment effect, restaging after treatment, and surveillance of early recurrence. PET is also an important research tool to map normal human brain and heart function, and to support drug development.

\section{PET imaging in oncology}

PET-CT imaging has been widely used in oncology. The most widely used tracer for PET-CT scans is fluorine-18 (F-18) fluorodeoxyglucose (FDG), called FDGPET-CT. This tracer is a glucose analog that is taken up by glucose-using cells and phosphorylated by hexokinase (whose mitochondrial form is greatly elevated in rapidly growing malignant tumors). Many evidences have shown that FDG-PET can be used for diagnosis, staging, and monitoring the treatment of cancers, particularly in Hodgkin's lymphoma, Non-Hodgkin's lymphoma [4], lung cancer [5], and other solid tumors [4]. The most useful application of FDG PET-CT for cancer patients is could detect recurrence, especially in asymptomatic patients with rising tumor marker levels and those with negative or equivocal conventional imaging findings [6].

A few other isotopes and radiotracers are slowly being introduced into oncology for specific purposes. For example, [11C] Metomidate has been used to detect tumors of adrenocortical origin [7].

\section{PET neuro-imaging}

PET imaging is also used for some brain diseases, because brain tumors, strokes, and neuron-damaging diseases which cause dementia (such as Alzheimer's disease) all cause great changes in brain metabolism, which in turn causes easily detectable changes in PET scans. For example, it is useful in early cases of Alzheimer's disease [8], where the early damage makes too little difference in brain volume and gross structure to change CT and standard MRI images enough to be able to reliably differentiate it from the "normal" area in brain.

\section{PET imaging in cardiology}

Now there are a number of tracers for PET scanning to assess myocardial viability. F-18 FDG is the tracer almost universally employed for clinical purposes. A study showed that FDG PET could predict improvement in left ventricular (LV) function. Its positive predictive value in this study was $86 \%$, negative predictive value was $100 \%$, and accuracy was $90 \%$ [9].

\section{PET imaging in infectious diseases}

PET has been widely used clinically to image bacterial infections by using FDG to identify the infection-associated inflammatory response. Recently, three different PET contrast agents have been developed to image bacterial infections in vivo: [18F] maltose [10], [18F] maltohexaose, and [18F] 2-fluorodeoxysorbitol (FDS) [11]. FDS also has the added benefit of being able to target only Enterobacteriaceae.

\section{PET/MR is on the way}

MRI is an innovative technique in the diagnostic imaging field. It can provide images of the body in many different planes, and represents an extraordinary addition to our diagnostic tools. MRI is particularly useful for the scanning and detection of abnormalities in soft tissue structures in the body, like cartilage and soft organs like the brain or heart. There is no involvement of any kind of radiation in MRI, so it is safe for people who can be vulnerable to the effects of radiation such as pregnant women or babies. Therefore, the 
combination of PET and MR imaging systems is the long lasting dream of many scientists. This will form a powerful new imaging modality.

Hans F. Wehrl [12] wrote that PET-MR technology is becoming more and more mature. It has great potential in preclinical and clinical research. For animal research, he predicted that PET-MR will replace combined PET-CT in the near future because of its inherent strengths such as soft-tissue contrast, multifunctional imaging capabilities, and the lack of ionizing radiation.

\section{PET imaging in pharmacokinetics}

In preclinical trials, PET imaging could facilitate the process of new drug design and biodistribution studies in animals, since it does not require dissecting the animals to discover the same information. The data from PET studies is easily interpreted in pharmacological terms and can be readily incorporated into the drug development process. This PET-derived information is so informative that it can be used to make go/no-go decisions, and has therefore become a routine investigational technique considered by pharma and biotech in developing central nervous system (CNS) therapeutics [13]. Due to the advent of PET imaging technology, a pharmacological company could save a large quantity of money and time. However, the lack of specific radioligands for many diseases still remains a large challenge for PET molecular imaging, both for academic research into understanding disease and for application to drug development.

\section{The side effect of PET imaging}

Up to date, there are no significantly known side effects to having a PET scan performed sine this imaging technology has been used clinically, which is very similar to a regular injection. The main issue of PET scan to worry about for the patients is the radiation exposure of the radiotracer, such as 18F-2-fluoro-2-deoxy-D-glucose (FDG). Actually, the dose of radiation of PET scan is similar to one time X-ray chest examination. So this is not a big issue for PET imaging.

In conclusion, PET-CT and PET-MR are playing more and more important roles in preclinical and clinical studies, and in patient diagnosis and treatment.

\section{References}

1. Bailey DL, Townsend DW, Valk PE, Maisey MN (2005) Positron Emission Tomography Basic Sciences.

2. Ter-Pogossian MM, Phelps ME, Hoffman EJ, Mullani NA (1975) A positron-emission transaxial tomograph for nuclear imaging (PETT). Radiology 114: 89-98.

3. Phelps ME, Hoffman EJ, Mullani NA, Ter-Pogossian MM (1975) Application of annihilation coincidence detection to transaxial reconstruction tomography. Journal of Nuclear Medicine 16: 210-224.

4. Dhanapathi H, Kumar R (2007) F-18 FDG PET/PET-CT in the management of lymphoma. Indian J Med Pediatr Oncol 28: 17-23.

5. Miele E, Spinelli GP, Tomao F, Zullo A, De Marinis F, et al. (2008) Positron Emission Tomography (PET) radiotracers in oncology--utility of 18F-Fluoro-deoxy-glucose (FDG)-PET in the management of patients with non-small-cell lung cancer (NSCLC). J Exp Clin Cancer Res. 27: 52.

6. Almuhaideb A, Papathanasiou N, Bomanji J (2011) 18F-FDG PET/CT imaging in oncology. Ann Saudi Med 31: 3-13.

7. Minn H, Salonen A, Friberg J, Roivainen A, Viljanen T, et al. (2004) Imaging of adrenal incidentalomas with PET using (11)C-metomidate and (18)F-FDG. J Nucl Med 45: 972-979.

8. Catafau AM, Bullich S (2015) Amyloid PET imaging: applications beyond Alzheimer's disease. Clin Transl Imaging 3: 39-55.

9. Schmidt M, Voth E, Schneider CA ( 2004) F-18-FDG uptake is a reliable predictory of functional recovery of a kinetic but viable infarct regions as defined by magnetic resonance imaging before and after revascularization. Magn Reson Imaging 22: 229-236

10. Gowrishankar G, Namavari M, Jouannot EB, Hoehne A, Reeves R, et al. (2014) Investigation of 6-[18F]-fluoromaltose as a novel PET tracer for imaging bacterial infection. PLoS One 9: e107951.

11. Weinstein EA, Ordonez AA, DeMarco VP, supriya P, Murawski AM et al. (2014) Imaging Enterobacteriaceae infection in vivo with $18 \mathrm{~F}$ fluorodeoxysorbitol positron emission tomography. Science Translational Medicine $6: 259$ ra146

12. Wehrl HF, Sauter AW, Divine MR, Pichler BJ (2015) Combined PET/MR: a technology becomes mature. J Nucl Med 56: 165-168.

13. Jones T1, Rabiner EA (2012) The development, past achievements, and future directions of brain PET. J Cereb Blood Flow Metab 32: 1426-1454. 